\title{
EULERIAN FORMULATION USING LAGRANGIAN MARKER PARTICLES WITH REFERENCE MAP TECHNIQUE FOR FLUID-STRUCTURE INTERACTION PROBLEM
}

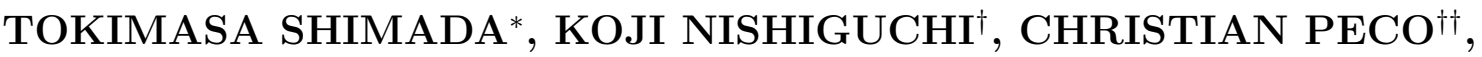 SHIGENOBU OKAZAWA ${ }^{\dagger \dagger}$ AND MAKOTO TSUBOKURA ${ }^{\dagger+\dagger}$}

\author{
*, $\dagger \dagger \dagger$ Graduate School of System Informatics, Kobe University \\ 1-1 Rokkodai-cho, Nada-ku, Kobe, Hyogo, 657-8501, JAPAN \\ RIKEN Center for Computational Science, RIKEN \\ 7-1-26 Minatojima-minami-machi, Chuo-ku, Kobe, Hyogo, 650-0047, JAPAN \\ e-mail: *shimada_t@stu.kobe-u.ac.jp, ${ }^{\dagger \dagger \dagger}$ tsubo@tiger.kobe-u.ac.jp \\ $\dagger$ Department of Civil Engineering, Nagoya University \\ Furo-cho, Chikusa-ku, Nagoya, Aichi, 464-8603, JAPAN \\ e-mail: kojinishiguchi@civil.nagoya-u.ac.jp \\ †† Engineering Science and Mechanics, Pennsylvania State University \\ University Park, 16802, USA \\ e-mail: christian.peco@psu.edu \\ t†† Faculty of Engineering, University of Yamanashi \\ 4-3-11 Takeda, Kofu, Yamanashi, 400-8511, JAPAN \\ e-mail: sokazawa@yamanashi.ac.jp
}

Key words: Fluid-Structure Interaction, Eulerian Method, Eulerian Unified Formulation, Finite Volume Method, Reference Map Technique

\begin{abstract}
Full Eulerian methods constitute a family of numerical techniques used to simulate fluid-structure interaction problems. In a full Eulerian method, the velocity gradient tensor is used to compute deformation of solid. However, it is difficult to compute solid stress accurately near the interface, where the velocity between fluid and solid changes drastically. In this work, we propose an Eulerian formulation for fluid-structure interaction problems using Lagrangian marker particles with the Reference Map Technique to compute the deformation of solid accurately near material interfaces without using the gradient of the velocity. We illustrate and validate the proposed method through the presentation of various benchmark problems.
\end{abstract}




\section{INTRODUCTION}

Full Eulerian methods[1, 2, 3] using a fixed mesh to compute motions of fluids and solids have been developed to solve fluid-structure interaction (FSI) problems. These methods are suitable for high-performance computing and computing large deformation of solids. In these methods, color functions like the volume-of-fluid (VOF) method[4] are used to express spatial distribution and shape of materials. Moreover, advection equations are used to compute not only color functions but also internal variables of solid. However, the numerical dissipation of color functions and internal variables of solid is unavoidable due to solving the advection equations in the Eulerian way[3].

We have introduced Lagrangian marker particles into the full Eulerian FSI formulation proposed by Nishiguchi et al.[3] to avoid solving advection equations[5]. In this method, Lagrangian marker particles represent solid regions and have internal variables of solids. We have confirmed our method obtains better accuracy results than results by the conventional Eulerian method. However, it is difficult to compute solid stress accurately near the interface with our method, when the velocity near the interface between fluid and solid changes drastically. The reason is that the velocity gradient tensor is used to compute solid stress in our method.

The Reference Map Technique (RMT) [6] is one of the methods to compute solid stress

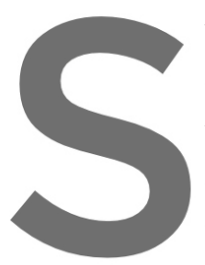
with a full Eulerian method. In the RMT,
distribution of the initial position vector (t
tion) of solid is used to compute the deform
can overcome the problem about the compu
using a velocity gradient tensor. However, in
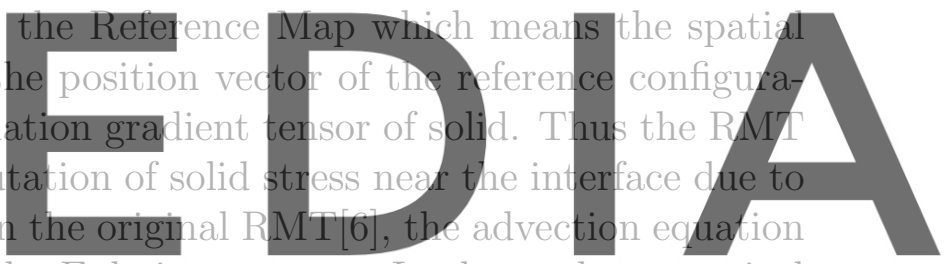

is solved to update the Reference Map in the Eulerian manner. In short, the numerical

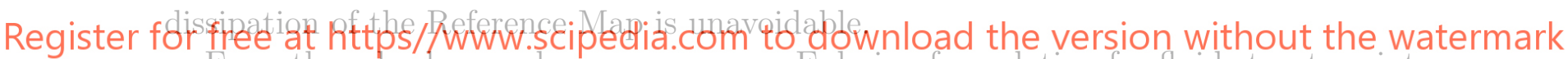

From these backgrounds, we propose an Eulerian formulation for fluid-structure interaction problems using Lagrangian marker particles with the Reference Map Technique to compute solid stress accurately without using the advection equation of the Reference map. We have confirmed that the proposed method overcomes the problem mentioned above. We explain our proposed method in this paper, but we are going to show the results of benchmark tests in the presentation.

\section{BASIC EQUATIONS}

\subsection{Mixture equations}

In this research, the incompressible mixture equations $[1,2,3]$ shown below are used to compute the motion of incompressible fluids and incompressible solids.

$$
\begin{gathered}
\nabla \cdot \boldsymbol{v}_{\text {mix }}=0 \\
\frac{\partial \rho_{\text {mix }} \boldsymbol{v}_{\text {mix }}}{\partial t}+\nabla \cdot\left(\rho_{\text {mix }} \boldsymbol{v}_{\text {mix }} \otimes \boldsymbol{v}_{\text {mix }}\right)=\nabla \cdot \boldsymbol{\sigma}_{\text {mix }}+\rho_{\text {mix }} \boldsymbol{b}
\end{gathered}
$$


Equation (1) is the mixture equation of continuity and equation (2) is the mixture equation of motion. In these equations, $\boldsymbol{v}_{\text {mix }}$ means the mixture velocity, $\boldsymbol{\rho}_{\text {mix }}$ means the mixture density, $\boldsymbol{\sigma}_{\text {mix }}$ means the mixture stress, and $\boldsymbol{b}$ is the body force. The single velocity field and pressure field are computed with these equations. In short, the velocity and pressure of each material are not obtained.

\subsection{Constitutive equations}

In this research, in order to compare the results of the benchmark problems by Zhao et al.[7] and by Nishiguchi et al.[3] with the result by the proposed method we use the same constitutive equations in their papers.

The constitutive equation of the fluid is an incompressible Newtonian fluid,

$$
\sigma=2 \mu \boldsymbol{D}-p \boldsymbol{I},
$$

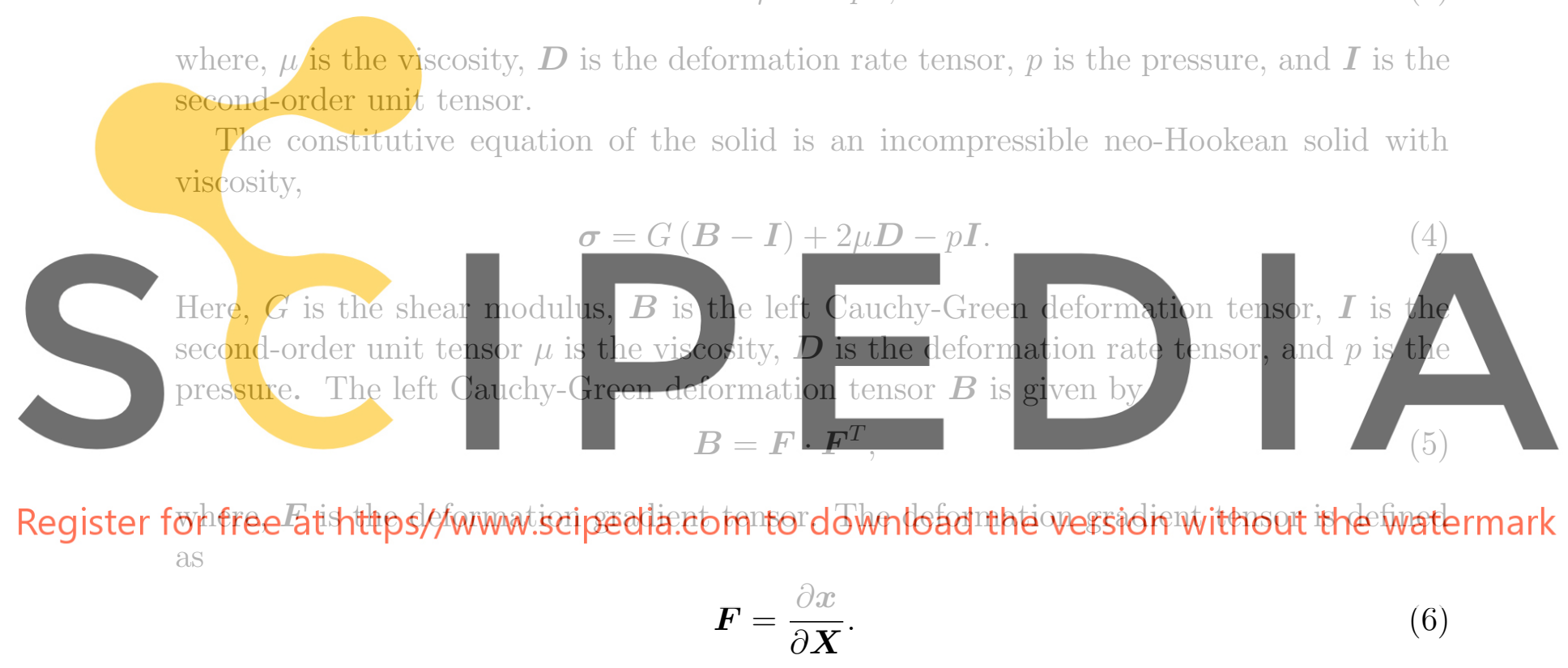

Here, $\boldsymbol{x}$ is the position vector of the current configuration and $\boldsymbol{X}$ is the position vector of the reference configuration.

\section{NUMERICAL METHOD}

Following our previous method[5], the governing equations and spatial differentials are solved on an Eulerian mesh and the physical quantities of solid are calculated and carried on Lagrangian marker particles in our proposed method in this paper.

The difference between the method in our previous paper and the proposed method is in the evaluation method of the left Cauchy green deformation tensor. In our previous method[5], the time evolution equation of the left Cauchy-Green deformation tensor is calculated on Lagrangian marker particles using the velocity gradient tensor calculated on the Eulerian mesh. However, in the proposed method, the deformation gradient tensor is 
obtained on a Euler grid using the Reference Map Technique[6], and then the left CauchyGreen deformation tensor is computed. In the proposed method, instead of solving the advection equation of the Reference Map, Lagrangian marker particles carry the Reference Map to prevent the numerical dissipation of the Reference Map.

Based on our previous method[3, 5], the finite volume method and the fractional step method[8] was used to solve the governing equations $(1,2)$ on the Eulerian mesh. The mixture velocity and pressure are computed at cell centers of the Eulerian mesh by a collocated variable arrangement, and density and volume fraction are also defined at the cell centers. The deviation stress of an incompressible neo-Hooke solid is defined at cell faces of the Eulerian mesh, while the deviation stress of the viscosity of a fluid and a solid is defined at the cell centers. In addition, the Reference Map $\boldsymbol{X}$ and the deformation gradient tensor $\boldsymbol{F}$ are defined at the cell centers.

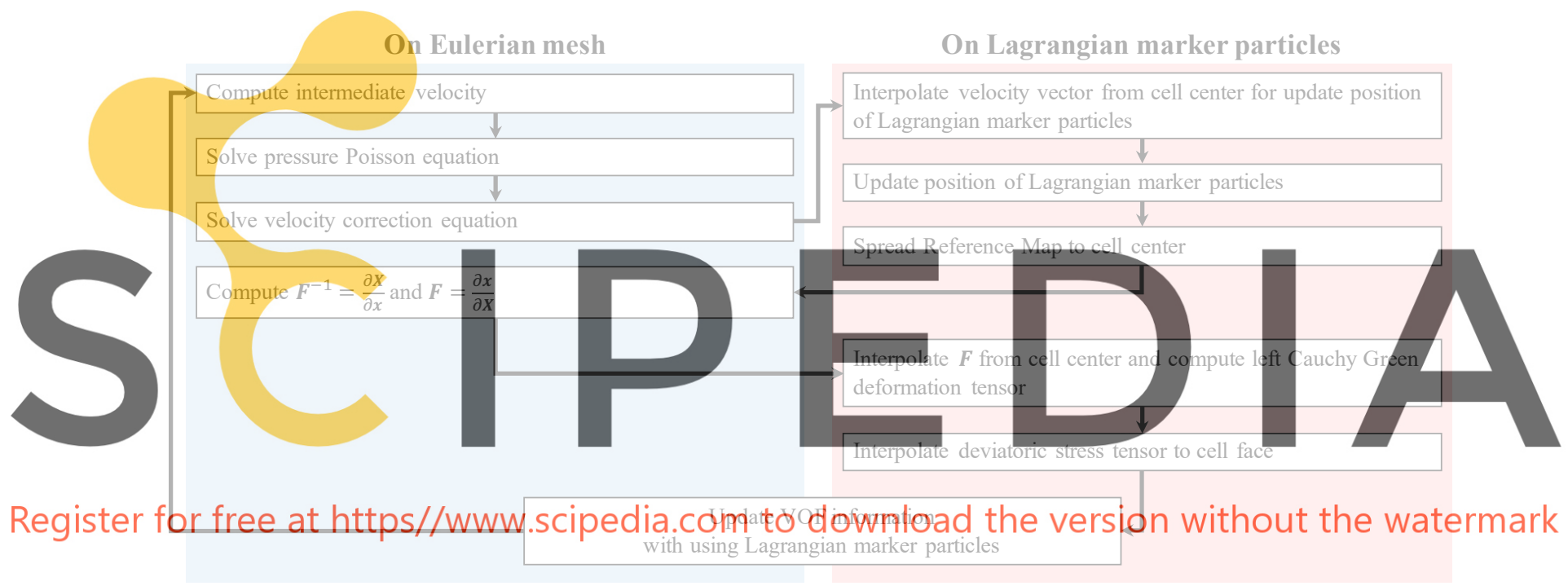

Figure 1: Computational flowchart of the proposed method

\subsection{Computation of intermediate velocity}

As described above, to solve the governing equation with the fractional step method, the intermediate velocity $\boldsymbol{v}_{\text {mix }}^{*}$ is calculated based on the following equation.

$$
\rho_{\text {mix }}^{n} \frac{\boldsymbol{v}_{\text {mix }}^{*}-\boldsymbol{v}_{\text {mix }}^{n}}{\Delta t}+\nabla \cdot\left(\rho_{\text {mix }} \boldsymbol{v}_{\text {mix }} \otimes \boldsymbol{v}_{\text {mix }}\right)=\nabla \cdot \boldsymbol{\sigma}_{\text {mix }}^{\prime}+\rho_{\text {mix }} \boldsymbol{b}
$$

In this study, the second-order Adams-Bashforth method is used for the time integration scheme of the advection term and stress term and the second-order central difference scheme is used for the spatial discretization scheme of the advection term. 


\subsection{Computation of pressure Poisson equation}

Next, the pressure $p^{n+1}$ at the next time step $(n+1)$ is obtained by solving the following pressure Poisson equation using the intermediate velocity $\boldsymbol{v}_{\mathrm{mix}}^{*}$.

$$
\frac{\nabla \cdot \boldsymbol{v}_{\mathrm{mix}}^{*}}{\Delta t}=\nabla \cdot\left(\frac{1}{\rho_{\mathrm{mix}}^{n}} \nabla p^{n+1}\right)
$$

Equation (8) is discretized with the second-order central difference scheme. The discretized equation is solved using the successive overrelaxation method color-coded by red and black ordering[9]. It is known that a collocated variable arrangement cannot avoid checkerboard pressure instability occurs because a pressure difference between adjacent cells cannot be evaluated. Thus, in this study, the Rhie-Chow method[10] is used to refrain from the pressure instability.

\subsection{Computation of velocity correction equation}

Finally, the velocity $\boldsymbol{v}_{\text {mix }}^{n+1}$ at the next time step $(n+1)$ is obtained by solving the velocity correction equation which is given by
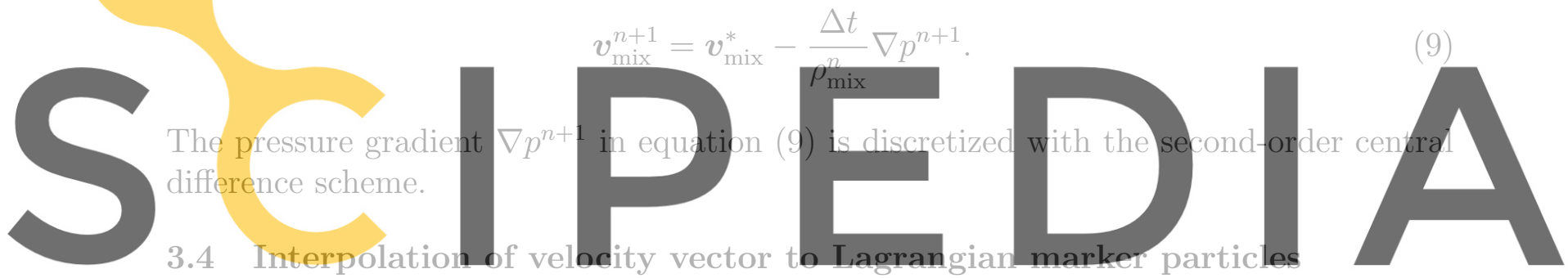

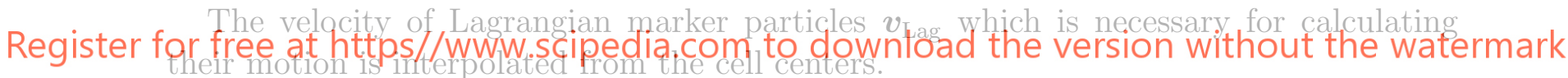

\subsection{Ujpdate position of Lagrangian marker particies}

The position of each Lagrangian marker particle in the next step $\boldsymbol{x}_{\mathrm{Lag}}^{n+1}$ is discretized with the second-order Adams-Bashforth method and given by

$$
\boldsymbol{x}_{\mathrm{Lag}}^{n+1}=\boldsymbol{x}_{\mathrm{Lag}}^{n}+\Delta t\left(\frac{3}{2} \boldsymbol{v}_{\mathrm{Lag}}^{n}-\frac{1}{2} \boldsymbol{v}_{\mathrm{Lag}}^{n-1}\right) \text {. }
$$

\subsection{Interpolation Reference Map to Eulerian mesh}

The Reference Map is interpolated from the Lagrangian marker particles to the cell centers. Since the Reference Map is the position vector of the reference configuration of solid, the position vector at the initial computational step is uniquely assigned to each Lagrangian marker particle. 


\subsection{Computation of deformation gradient tensor}

Using the Reference Map value $\boldsymbol{X}$ interpolated to the cell centers, the inverse tensor of the deformation $\boldsymbol{F}^{-1}$ gradient tensor is obtained as follows.

$$
\boldsymbol{F}^{-1}=\frac{\partial \boldsymbol{X}}{\partial \boldsymbol{x}}
$$

In our method, the inverse of the deformation gradient tensor is defined at the cell centers and calculated using the second-order central difference. Finally, the deformation gradient tensor at the cell centers $\boldsymbol{F}$ is obtained by calculating the inverse of $\boldsymbol{F}^{-1}$ obtained by the above equation (11).

\subsection{Interpolation of deformation gradient tensor}

The deformation gradient tensor $\mathbb{F}$ computed on the cell centers is interpolated to the Lagrangian marker particles.

\subsection{Computation of solid deviation stress and interpolation to the cell face}

Using the deformation gradient tensor $\boldsymbol{F}$ interpolated to the Lagrangian marker particles, the left Cauchy green deformation tensor is obtained on each Lagrangian marker particle from equation (5). After that the solid deviation st
right hand side of equation (4) is calculated on the Lagrang
the deviation stress on Lagr ngian marker particles are inter
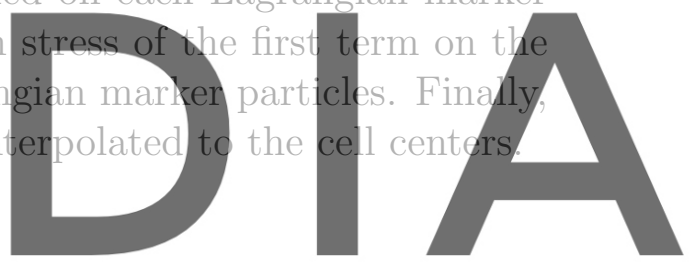

At the end of the computation in one time step, the volume fraction of solids in each

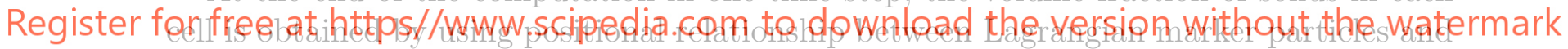
each cell.

\section{NUMERICAL EXAMPLES}

To verify the proposed method, we conducted the numerical benchmark tests by Zhao et al.[7] and by Nishiguchi et al.[3] The results of them are going to be shown in the presentation.

\section{CONCLUSIONS}

In this paper, we proposed the Eulerian formulation method for FSI problems using the Reference Map Technique and Lagrange marker particles to solve the problem that stress oscillation occurs near an interface of materials. In order to confirm the effectiveness and validity of the proposed method, the numerical benchmark tests[7] have been conducted. 


\section{REFERENCES}

[1] Sugiyama, K., Ii, S., Takeuchi, S., Takagi, S., and Matsumoto, Y. A full Eulerian finite difference approach for solving fluid-structure coupling problems. Journal of Computational Physics. (2011) 230-3:596-627

[2] Nishiguchi, K., Okazawa, S., and Tsubokura, M. Multimaterial Eulerian finite element formulation for pressure-sensitive adhesives. International Journal for Numerical Methods in Engineering. (2018) 114-13:1368-1388

[3] Nishiguchi, K., Bale, R., Okazawa, S., and Tsubokura, M. Full Eulerian deformable solid-fluid interaction scheme based on building-cube method for large-scale parallel computing. International Journal for Numerical Methods in Engineering. (2019) 117-2:221-248

[4] Hirt, C. W.and Nichols, B. D. Volume of fluid (VOF) method for the dynamics of free boundaries. Journal of computational physics. (1981) 39-1:201-225

[5] Shimada, T., Nishiguchi, K., Bale, R., Okazawa, S., and Tsubokura, M. Eulerian finite volume formulation using Lagrangian marker particles for incompressible fluid-
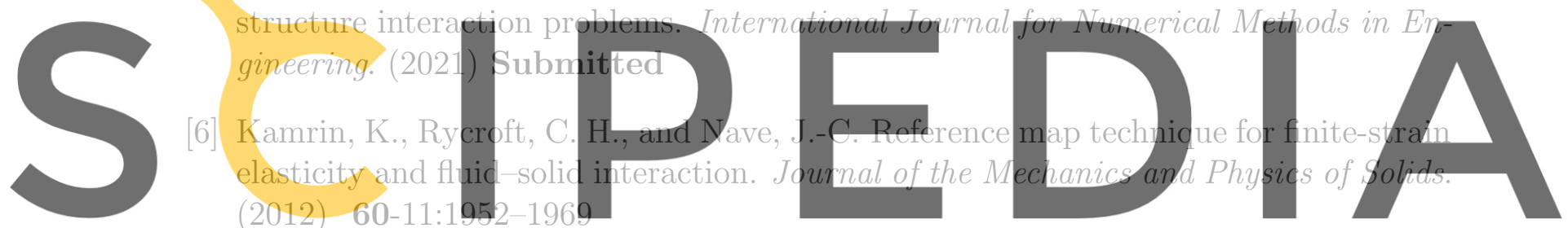

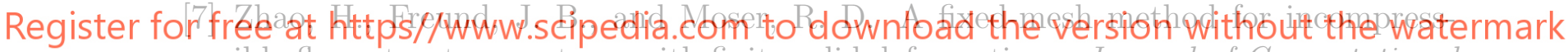
ible flow-structure systems with finite solid deformations. Journal of Computational Physics. (2008) 227-6:3114-3140

[8] Kim, J.and Moin, P. Application of a fractional-step method to incompressible Navier-Stokes equations. Journal of computational physics. (1985) 59-2:308-323

[9] Yavneh, I. On red-black SOR smoothing in multigrid. SIAM Journal on Scientific Computing. (1996) 17-1:180-192

[10] Rhie, C.and Chow, W. L. Numerical study of the turbulent flow past an airfoil with trailing edge separation. AIAA journal. (1983) 21-11:1525-1532 